Check for updates

Cite this: RSC Adv., 2019, 9, 25377

Received 26th June 2019

Accepted 2nd August 2019

DOI: $10.1039 / c 9 r a 04836 g$

rsc.li/rsc-advances

\title{
Chiral benzene backbone-based sulfoxide-olefin ligands for highly enantioselective Rh-catalyzed addition of arylboronic acids to $\mathbf{N}$-tosylarylimines $\uparrow$
}

\author{
Feng Xue, (D) *a Qibin Liu, ${ }^{\text {tb }}$ Yong Zhu, ${ }^{a}$ Yunfei Qing ${ }^{a}$ and Boshun Wan (D) *c
}

An efficient Rh-catalyzed addition of arylboronic acids to $N$-tosylarylimines has been developed with chiral benzene backbone-based sulfoxide-olefin ligands, where 2-methoxy-1-naphthyl sulfinyl functionalized olefin ligands have shown to be more effective. The versatile method tolerates a wide range of functional groups and shows broad scope without regard to electronic or steric substitution pattern, allowing access to a broad range of chiral diarylmethylamines in high yields (up to 99\%) with excellent enantioselectivities (up to $99 \%$ ee).

\section{Introduction}

Optically active diarylmethylamines are present in a variety of biologically significant structures. ${ }^{1}$ Due to their pharmaceutical importance, great attention has been drawn to the development of their synthesis. ${ }^{2}$ Rhodium-catalyzed asymmetric addition of organoboron reagents to aldimines has become a powerful strategy for the straightforward synthesis of the skeleton since the first report by Tomioka using L-valine-connected amidomonophosphane as chiral ligand in $2004 .^{3}$ From then on, considerable efforts have been made and great progress has been achieved in the catalytic enantioselective arylation of $N$ tosyl or nosyl activated/protected imines. ${ }^{4}$ Among them, Hayashi and co-workers ${ }^{5}$ reported excellent enantioselectivities for the addition of aryl boroxines to $N$-tosylarylimines by employing chiral bicyclo[2.2.2]octadiene ligand. In 2006, Zhou and coworkers $^{6}$ also reported an efficient asymmetric arylation of $N$ tosylarylimines using monodentate spiro phosphite $(S)$-ShiP. In 2007 and 2010, Lin and co-workers ${ }^{7}$ successively reported other examples in the highly efficient arylation of $N$-tosylarylimines with chiral bicyclo-[3.3.0]octadiene and dicyclopentadienes ligands. In 2014, $\mathrm{Wu}$ and co-workers ${ }^{8}$ adopted another diene ligand in enantioselective Rh-catalyzed arylation of $N$-tosyl and $N$-nosyl aldimines in methanol. Later, Dorta and co-workers ${ }^{9}$ employed chiral disulfoxide ligand for the efficient rhodiumcatalyzed 1,2-addition of arylboroxines to $N$-tosylarylimines in

${ }^{a}$ Key Laboratory of Functional Organic Molecules of Xinxiang City Henan Province, College of Chemistry and Chemical Engineering, Henan Institute of Science and Technology, Xinxiang, Henan, 453002, China. E-mail: fxuehist@sina.com

${ }^{b}$ Dalian Allychem Co., Ltd, 5 Jinbin Road, Dalian 116620, China. E-mail: qliu@ allychem.com

'Dalian Institute of Chemical Physics, Chinese Academy of Sciences, 457 Zhongshan Road, Dalian 116023, P. R. China. E-mail: bswan@dicp.ac.cn

$\dagger$ Electronic supplementary information (ESI) available. See DOI: 10.1039/c9ra04836g
2016. Recently, Lin and co-workers applied chiral spiro monophosphite-olefin ligands in asymmetric addition of organoboronic acids to aldimines. ${ }^{10}$ Despite the remarkable advances with these ligands (Scheme 1a), the substrate generality is likely influenced by the electronic and steric substitution pattern of both reaction partners to show relatively narrow compatibility of

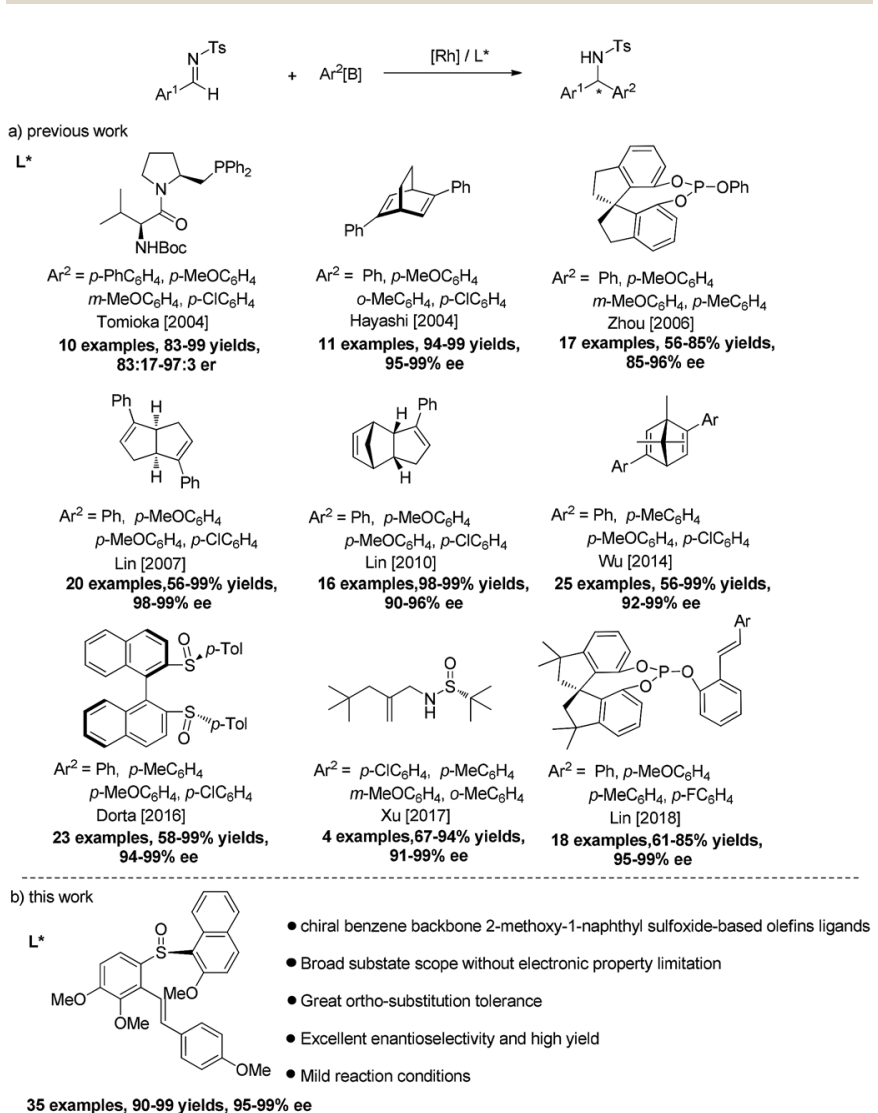

Scheme 1 Rh-catalyzed asymmetric arylation of $N$-tosylarylimines. 
reaction substrates. For example, these methods mainly focused on less sterically hindered para- as well as electron-rich substitution arylboron reagents. Moreover, limited research has been conducted on substrates with electron-poor groups and orthosubstituent groups. Thus, it is required to broaden the substrate scope of this Rh-catalyzed asymmetric arylation reaction with greater substitution tolerance.

With $o$-phenylene as the linkage of olefin and sulfoxide, $\mathrm{we}^{11}$ have successfully developed a series of simple and easily tunable chiral benzene backbone-based olefin-sulfoxide ligands bearing different olefin and sulfinyl moieties for the Rhcatalyzed enantioselective addition reaction, where 2-methoxy1-naphthyl sulfinyl functionalized olefin ligands ${ }^{11 b, c}$ have high efficiency for the conjugate addition of arylboronic acids to nitroalkenes and unsaturated esters. Meanwhile, other chiral sulfinyl-based olefin ligands (SOLs) ${ }^{12}$ have also been explored and applied in a range of Rh-catalyzed asymmetric transformations, which have obvious advantage over some conventional chiral ligands in terms of activity and selectivity. Recently, $\mathrm{Xu}$ and co-workers ${ }^{12 j}$ reported highly enantioselective addition of aryl boroxines to $N, N$-dimethylsulfamoyl-protected aldimines by employing chiral branched tert-butyl sulfonamide-based olefins ligands, in which limited examples were tentatively conducted on the reaction of $\mathrm{N}$-tosylarylimines with arylboroxines without systematically examined the reaction (Scheme 1a). Despite the significant progress, the sulfinyl moieties of the reported sulfinyl-based olefin ligands were mostly limited to tert-butyl substitution, and the incorporation of alkenes with other sulfinyl groups remains underappreciated and far less explored. In view of the pharmaceutical importance of chiral diarylmethylamines in organic transformations, it is still highly desirable to develop effective catalytic systems that would successfully lead to a broad range of desired diarylmethylamines with excellent enantioselectivities. To this end, we explored the asymmetric addition of aryl boronic acids to $\mathrm{N}$ tosylarylimines by using chiral benzene backbone-based sulfoxide-olefin ligands based on 2-methoxy-1-naphthyl sulfinyl moiety, affording a broad range of chiral diarylmethylamines in high yields (up to 99\%) with excellent enantioselectivities (up to $99 \%$ ee) (Scheme 1b).

\section{Results and discussion}

We started with Rh-catalyzed conjugate addition of $N$-tosylphenylimine 1a with $p$-anisylboronic acid $\mathbf{2 a}$ in the presence of ligands L1-L6 (Table 1). Initially, the reaction proceeded in the presence of $1.5 \mathrm{~mol} \%\left[\mathrm{RhCl}\left(\mathrm{C}_{2} \mathrm{H}_{4}\right)_{2}\right]_{2}$ and $3.3 \mathrm{~mol} \% \mathrm{L1}$ or L2a bearing tertbutylsulfinyl moiety in $\mathrm{Et}_{3} \mathrm{~N} /$ toluene, giving a trace amount of desired product (entries 1-2). To improve the activity, ligands L2bL2d bearing different sulfinyl moieties were screened. It was found that ligand $\mathbf{L} 2 \mathbf{b}$ with $p$-tolsulfiny moiety gave the expected product 3aa in $75 \%$ yield with $40 \%$ ee (entry 3 ), whereas ligand L2c bearing $p$-methoxybenzene sulfinyl moieties gave $60 \%$ ee (entry 4 ) and L2d with 2-methoxy-1-naphthyl sulfinyl moiety afforded higher yield (85\%) and enantioselectivity (76\% ee) (entry 5). With 2-methoxy-1naphthyl moiety was established, ligands L3-L6 bearing different alkene moieties containing substituents with different steric and
Table 1 Screening of ligands, solvents, and bases in the addition reaction $^{a}$

End
Entry

${ }^{a}$ The reaction was carried out with $N$-tosylphenylimine $1 \mathrm{a}(0.30 \mathrm{mmol})$, $p$-anisylboronic acid $2 \mathrm{a}(0.45 \mathrm{mmol}),\left[\operatorname{RhCl}\left(\mathrm{C}_{2} \mathrm{H}_{4}\right)_{2}\right]_{2}(0.0045 \mathrm{mmol})$, ligand (0.0099 mmol, 1.1 equiv. to $\mathrm{Rh})$, and $0.75 \mathrm{M} \mathrm{Et}_{3} \mathrm{~N}(0.20 \mathrm{~mL})$ in toluene $(2.0 \mathrm{~mL})$ at $50{ }^{\circ} \mathrm{C}$ for $5-6 \mathrm{~h}$. ${ }^{b}$ Yield based on $N$ tosylphenylimine. ${ }^{c}$ Determined by HPLC analysis. ${ }^{d}$ Not determined.

electronic natures were further examined, meanwhile, the substitution effects on the central benzene ring moiety were also explored (entries 6-11). It was speculated that the electronic property of the substitutes at the olefin moiety would have a significant effect on the enantioselectivity. Ligand L3f possessing an electron-donating para-methoxy group on the terminal benzene ring afforded the product with higher enantioselectivity (entry $7 \mathrm{vs.}$ entries 6 and 8). Moreover, the electronic effect on the benzene backbone was also examined. Ligands L4f bearing a MOMO substituent on the benzene backbone showed similar reactivity as that of L3f (entry $9 v$ s. entry 7). However, when an additional methoxy group was introduced to the ortho-position of $\mathrm{MeO}$ on the benzene backbone of $\mathbf{L 3 f}$, ligand L6f afforded the product with increased catalytic reactivity and enantioselectivity ( $92 \%$ yield, $95 \%$ ee, entry 11) while ligand L5f showed inferior result ( $80 \%$ ee, entry 10).

Next, the influence of solvents and bases was further explored (Table 2). Other inorganic bases such as $\mathrm{KOH}, \mathrm{K}_{2} \mathrm{CO}_{3}$, and $\mathrm{K}_{3} \mathrm{PO}_{4}$ did not improve the enantioselectivity (entries 1-3). When the reaction was conducted in aqueous $\mathrm{KHF}_{2} /$ toluene solution, in which potassium aryltrifluoroborate can be generated in situ ${ }^{13}$ no increase of reactivity and enantioselectivity was also observed (entry 4). Gratifyingly, KF afforded highest enantioselectivity ( $97 \%$ ee, entry 5). Furthermore, solvent screening showed that dioxane, THF, DCE and $\mathrm{CH}_{2} \mathrm{Cl}_{2}$ failed to improve the enantioselectivity (entries 6-9). In addition, other 
Table 2 Screening of ligands, solvents, and bases in the addition reaction $^{a}$

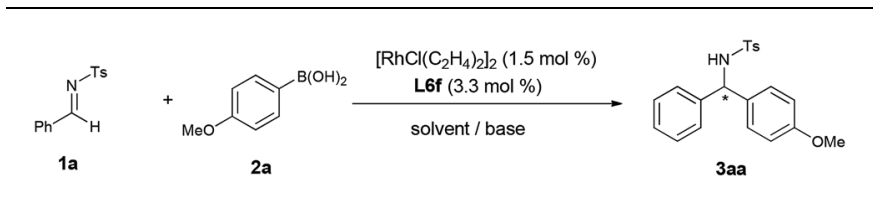

\begin{tabular}{|c|c|c|c|c|}
\hline Entry & Solvent & Base & Yield $^{b}(\%)$ & $\mathrm{ee}^{c}(\%)$ \\
\hline 1 & Toluene & КОН (0.75 M) & 95 & 91 \\
\hline 2 & Toluene & $\mathrm{K}_{2} \mathrm{CO}_{3}(1.5 \mathrm{M})$ & 90 & 92 \\
\hline 3 & Toluene & $\mathrm{K}_{3} \mathrm{PO}_{4}(1.5 \mathrm{M})$ & 90 & 92 \\
\hline 4 & Toluene & $\mathrm{KHF}_{2}(1.5 \mathrm{M})$ & 90 & 93 \\
\hline 5 & Toluene & $\mathrm{KF}(1.5 \mathrm{M})$ & 96 & 97 \\
\hline 6 & Dioxane & $\mathrm{KF}(1.5 \mathrm{M})$ & 91 & 91 \\
\hline 7 & $\mathrm{THF}$ & $\mathrm{KF}(1.5 \mathrm{M})$ & 93 & 91 \\
\hline 8 & DCE & $\mathrm{KF}(1.5 \mathrm{M})$ & 92 & 89 \\
\hline 9 & $\mathrm{CH}_{2} \mathrm{Cl}_{2}$ & $\mathrm{KF}(1.5 \mathrm{M})$ & 91 & 96 \\
\hline $10^{d}$ & Toluene & $\mathrm{KF}(1.5 \mathrm{M})$ & 94 & 97 \\
\hline $11^{e}$ & Toluene & $\mathrm{KF}(1.5 \mathrm{M})$ & 93 & 95 \\
\hline
\end{tabular}

${ }^{a}$ The reaction was carried out with $N$-tosylphenylimine $1 \mathrm{a}(0.30 \mathrm{mmol})$, $p$-anisylboronic acid $2 \mathrm{a}(0.45 \mathrm{mmol}),\left[\operatorname{RhCl}\left(\mathrm{C}_{2} \mathrm{H}_{4}\right)_{2}\right]_{2}(0.0045 \mathrm{mmol})$, ligand L6f $(0.0099 \mathrm{mmol}, 1.1$ equiv. to $\mathrm{Rh})$, and $1.5 \mathrm{M}$ aq base $(0.20$ $\mathrm{mL})$ in solvent $(2.0 \mathrm{~mL})$ at $50{ }^{\circ} \mathrm{C}$ for $5-6 \mathrm{~h}$. ${ }^{b}$ Yield based on $N$ tosylphenylimine. ${ }^{c}$ Determined by HPLC analysis. ${ }^{d} p$-Methoxy phenylboroxine instead of $p$-anisylboronic acid. ${ }^{e} N$-Nosylphenylimine instead of $N$-tosylphenylimine.

organoboron reagents and aldimines such as $p$-methoxy phenylboroxine and $N$-nosylphenylimine exhibited comparable results as those of $p$-anisylboronic acid $\mathbf{2 a}$ and $N$-tosylphenylimine 1a (entries 10-11).

Having established the optimal reaction conditions, we then investigated the substrate scope of this Rh-catalyzed asymmetric arylation (Table 3 ). We were pleased to find that a variety of arylboronic acids bearing substituents with diverse electronic and steric properties all smoothly reacted with $\mathrm{N}$-tosylarylimines to provide the desired product in high yields (90-99\%) with excellent enantioselectivities (95-99\%). In general, the electronic properties of the substituent did not significantly affect the reaction stereoselectivity. Extremely high enantiomeric excesses (98-99\% ee) were attained with electron-poor or sterically encumbered arylboronic acids (entries 7-13 and 2628). Furthermore, a broad range of electronically and sterically different aryl imines were tested. Regardless of the substitution pattern on the phenyl ring, all these imines reacted well with arylboronic acids to provide the corresponding products in high yields with excellent enantioselectivity (95-99\% ee). Intriguingly, apart from almost same enantiocontrol happened in electron-poor imines $\mathbf{1 f}$ and arylboronic acids $2 \mathbf{i}$ (entry 9 vs. 20), significant enantiocontrol was observed with electron-rich imines; this trend is opposite to what is observed with arylboronic acids. Thus, in some cases such as for 1c and 1d, by simply reversing the corresponding $\mathrm{Ar}^{1}$ and $\mathrm{Ar}^{2}$ groups of the two substrates, the ee of the product can be readily enhanced (entry 1 vs. 18, entry 2 vs. 17, and also entry 32 vs. 24). These results indicate that the desired highly enantioenriched product could easily be furnished by switching the aryl acceptor/donor.
Table 3 Substrate scope in the addition reaction ${ }^{a}$

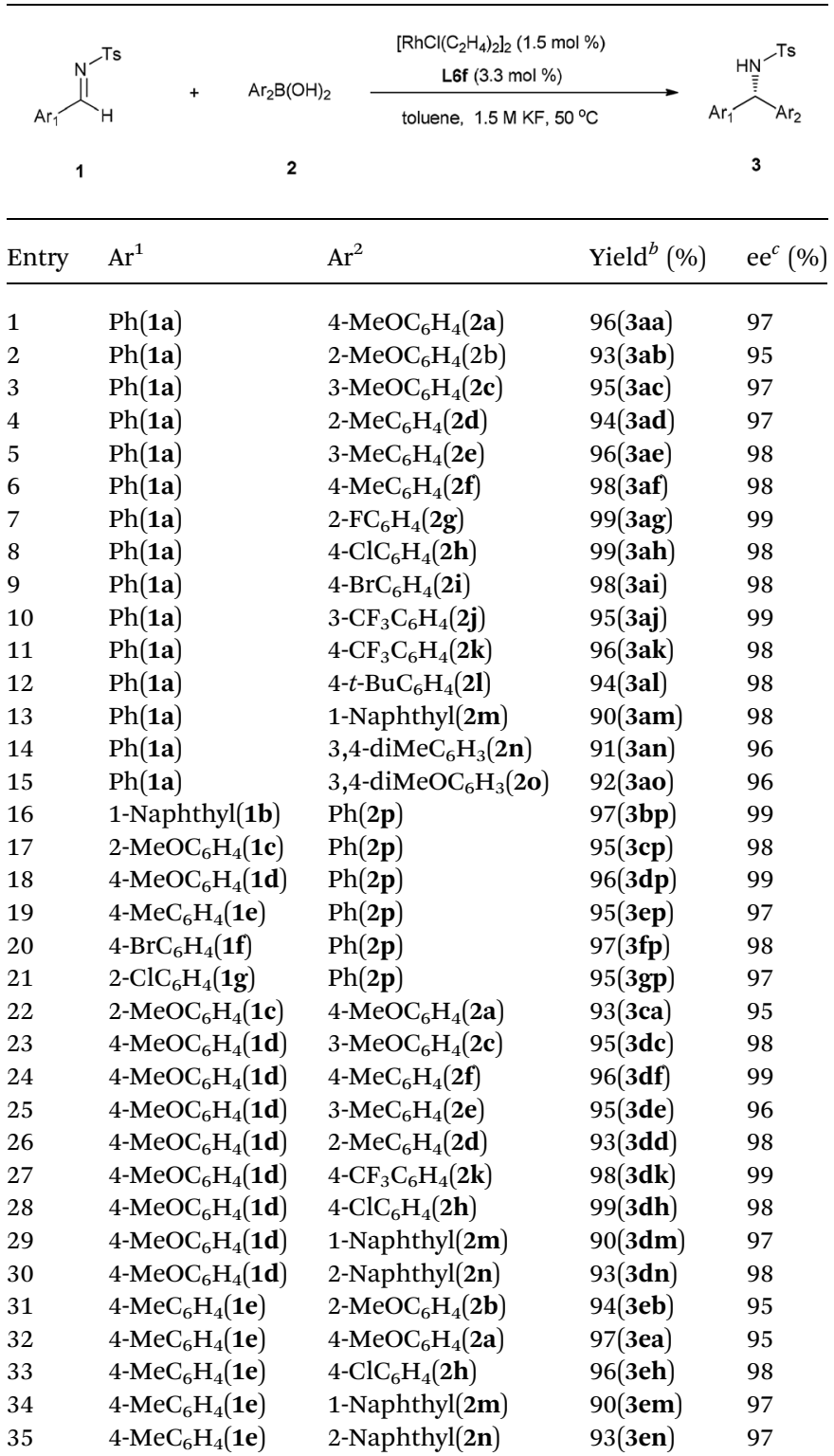

${ }^{a}$ The reaction was carried out with $N$-tosylarylimines $(0.30 \mathrm{mmol})$, arylboronic acids $(0.45 \mathrm{mmol}),\left[\mathrm{RhCl}\left(\mathrm{C}_{2} \mathrm{H}_{4}\right)_{2}\right]_{2}(0.0045 \mathrm{mmol})$, ligand L6f $(0.0099 \mathrm{mmol}, 1.1$ equiv. to $\mathrm{Rh})$, and $1.5 \mathrm{M}$ aq $\mathrm{KF}(0.20 \mathrm{~mL})$ in toluene $(2.0 \mathrm{~mL})$ at $50{ }^{\circ} \mathrm{C}$ for $5-6 \mathrm{~h}$. ${ }^{b}$ Yield based on $N$ tosylarylimines. ${ }^{c}$ Determined by HPLC analysis.

Another interesting feature is that the reaction exhibits a truly remarkable ortho-substitution tolerance. In all cases with sterically encumbered substrates, as exemplified by $\mathbf{2 d}$ (entry 4 ), $\mathbf{2 g}$ (entry 7 ), $\mathbf{2 m}$ (entry 13), 1b (entry 16), 1c (entry 17), $1 \mathrm{~g}$ (entry 21), nearly perfect enantiomeric excesses (97-99\% ee) could be achieved. In addition, it is worth mentioning that when it comes to arylboronic acids with $m$-OMe and $p$-Me groups on the phenyl ring, higher yield and enantioselecitivity were obtained than those in previous work $^{12 j}$ (entry 3, 95\% yield, 97\% ee $v s .67 \%$ yield, $94 \%$ ee and entry $6,98 \%$ yield, $98 \%$ ee $v s$. $94 \%$ yield, $91 \%$ ee).

On the basis of the reaction stereochemical outcome, an empirical transition state model ${ }^{\mathbf{1 4}}$ is proposed (Fig. 1). We 

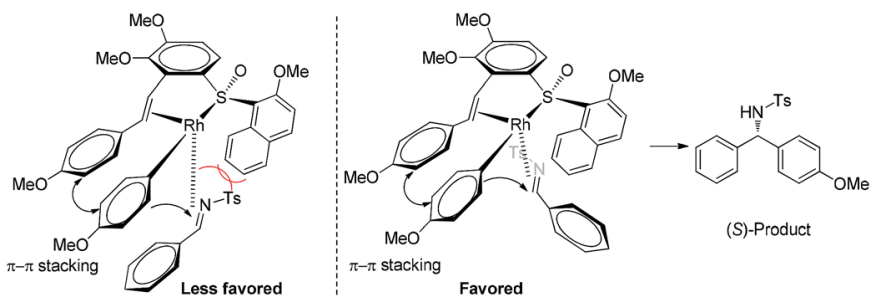

Fig. 1 Proposed stereochemical pathway for the asymmetric arylation.

assume that there tends to be a $\pi-\pi$ stacking between the phenyl ring of the ligand terminus and the metalated phenyl ring of phenylboronic acid in the reaction transition state. The Rh-complex recognizes the alkene moiety of $N$-tosylarylimine as a result of the steric repulsion between the 2-methoxy-1naphthyl group of the ligand and tosyl group of the $N$-tosylarylimine. To minimize steric congestion, coordination of $\mathrm{N}$ tosylarylimine from the less steric hindered side is more favorable, which leads to the $S$ isomer and is consistent with the observed stereochemistry.

\section{Conclusion}

In conclusion, an efficient Rh-catalyzed addition of arylboronic acids to $N$-tosylarylimines has been developed with chiral benzene backbone-based sulfoxide-olefin ligands, where 2methoxy-1-naphthyl sulfinyl functionalized olefin ligands have shown to be more effective than the tert-butyl sulfinyl-based ones, allowing access to a broad range of chiral diarylmethylamines in high yields (up to 99\%) with excellent enantioselectivities (up to $99 \%$ ee). Compared with the methods in the literature, both the electron effect and steric effect of the substituent group of the reaction substrate have no obvious influence on the reaction results, especially when the substrates with strong electron-withdrawing group or ortho-hindrance were involved in the reaction. Excellent yield and enantioselectivity could be also achieved, indicating that the catalytic system has a wide range of reaction substrate tolerance. This study sets the stage for further exploration of these recently developed ligands in other asymmetric transformations and the development of other kinds of unique olefin ligands. Further studies are underway and will be reported in due course.

\section{Experimental}

\section{General}

All reactions were carried out under an atmosphere of nitrogen using the standard Schlenk techniques, unless otherwise noted. Solvents were dried and distilled by standard procedures. ${ }^{1} \mathrm{H}$ NMR and ${ }^{13} \mathrm{C}$ NMR spectra were recorded at room temperature in $\mathrm{CDCl}_{3}$ on $400 \mathrm{MHz}$ and $600 \mathrm{MHz}$ instrument with tetramethylsilane (TMS) as internal standard. Enantiomeric excess was determined by HPLC analysis, using chiral column described below in detail. Optical rotations were measured by polarimeter. Flash column chromatography was performed on silica gel (200-300 mesh). All reactions were monitored by TLC analysis.
General procedure for the enantioselective Rh-catalyzed addition of arylboronic acids to $\mathrm{N}$-tosylarylimines

Under nitrogen atmosphere, a mixture of $\left[\mathrm{RhCl}\left(\mathrm{C}_{2} \mathrm{H}_{4}\right)_{2}\right]_{2}$ (1.8 mg, $0.0045 \mathrm{mmol})$ and ligand L6f $(4.7 \mathrm{mg}, 0.0099 \mathrm{mmol})$ in $1 \mathrm{~mL}$ toluene was stirred at room temperature for $1 \mathrm{~h}$. At which time arylboronic acid $(0.45 \mathrm{mmol})$ was added, followed by $N$ tosylarylimines $(0.30 \mathrm{mmol})$, aqueous $\mathrm{KF}\left(1.5 \mathrm{M}\right.$ in $\mathrm{H}_{2} \mathrm{O}, 0.20$ $\mathrm{mL}, 0.30 \mathrm{mmol})$ and toluene $(1 \mathrm{~mL})$. The reaction was stirred at $50{ }^{\circ} \mathrm{C}$ for $5-6 \mathrm{~h}$. When the reaction was over, the reaction mixture was concentrated in vacuo and purified by silica gel flash column chromatography (petroleum ether/ethyl acetate as eluent) to afford the product.

\section{Conflicts of interest}

There are no conflicts to declare.

\section{Acknowledgements}

Financial support from the National Natural Science Foundation of China (21172218) is gratefully acknowledged.

\section{Notes and references}

1 For representative examples, see: (a) C. M. Spencer, D. Foulds and D. H. Peters, Drugs, 1993, 46, 1055; (b) S. N. Calderon, R. B. Rothman, F. Porreca, J. L. FlippenAnderson, R. W. McNutt, H. Xu, L. E. Smith, E. J. Bilsky, P. Davis and K. C. Rice, J. Med. Chem., 1994, 37, 2125; (c) M. J. Bishop and R. W. McNutt, Bioorg. Med. Chem. Lett., 1995, 5, 1311; (d) S. Sakurai, N. Ogawa, T. Suzuki, K. Kato, T. Ohashi, S. Yasuda, H. Kato and Y. Ito, Chem. Pharm. Bull., 1996, 44, 765.

2 For representative examples, see: (a) S. Kobayashi and H. Ishitani, Chem. Rev., 1999, 99, 1069; (b) C. Bolm, J. P. Hildebrand, K. Muñiz and N. Hermanns, Angew. Chem., Int. Ed., 2001, 40, 3284; (c) F. Schmidt, R. T. Stemmler, J. Rudolph and C. Bolm, Chem. Soc. Rev., 2006, 35, 454; (d) S. Kobayashi, Y. Mori, J. S. Fossey and M. M. Salter, Chem. Rev., 2011, 111, 2626.

3 M. Kuriyama, T. Soeta, X. Hao, Q. Chen and K. Tomioka, J. Am. Chem. Soc., 2004, 126, 8128.

4 For representative examples, see: (a) T. Hayashi, M. Kawai and N. Tokunaga, Angew. Chem., Int. Ed., 2004, 43, 6125; (b) Y. Otomaru, N. Tokunaga, R. Shintani and T. Hayashi, Org. Lett., 2005, 7, 307; (c) D. J. Weix, Y. L. Shi and J. A. Ellman, J. Am. Chem. Soc., 2005, 127, 1092; (d) Modern Rhodium-Catalyzed Organic Reactions, ed. P. A. Evans, Wiley-VCH, Weinheim, 2005; (e) M. Trincado and J. A. Ellman, Angew. Chem., Int. Ed., 2008, 47, 5623; (f) Z. Cui, H. J. Yu, R. F. Yang, W. Y. Gao, C. G. Feng and G. Q. Lin, J. Am. Chem. Soc., 2011, 133, 12394; (g) X. Gao, B. Wu, Z. Yan and Y. G. Zhou, Org. Biomol. Chem., 2016, 14, 55 . 
5 N. Tokunaga, Y. Otomaru, K. Okamoto, K. Ueyama, R. Shintani and T. Hayashi, J. Am. Chem. Soc., 2004, 126, 13584.

6 H. F. Duan, Y. X. Jia, L. X. Wang and Q. L. Zhou, Org. Lett., 2006, 8, 2567.

7 (a) Z. Q. Wang, C. G. Feng, M. H. Xu and G. Q. Lin, J. Am. Chem. Soc., 2007, 129, 5336; (b) C. Shao, H. J. Yu, N. Y. Wu, C. G. Feng and G. Q. Lin, Org. Lett., 2010, 12, 3820.

8 C. C. Chen, B. Gopula, J. F. Syu, J. H. Pan, T. S. Kuo, P. Y. Wu, J. P. Henschke and H. L. Wu, J. Org. Chem., 2014, 79, 8077.

9 G. Z. Zhao, G. Sipos, A. Salvador, A. Ou, P. C. Gao, B. W. Skelton and R. Dorta, Adv. Synth. Catal., 2016, 358, 1759.

10 H. Y. Shan, Q. X. Zhou, J. L. Yu, S. Q. Zhang, X. Hong and X. F. Lin, J. Org. Chem., 2018, 83, 11873.

11 (a) F. Xue, X. C. Li and B. S. Wan, J. Org. Chem., 2011, 76, 7256; (b) F. Xue, X. C. Li and B. S. Wan, J. Org. Chem., 2012, 77, 3081; (c) F. Xue, D. P. Wang, X. C. Li and B. S. Wan, Org. Biomol. Chem., 2013, 11, 7893.

12 For representative examples, see: (a) T. Thaler, L. N. Guo, A. K. Steib, M. Raducan, K. Karaghiosoff, P. Mayer and
P. Knochel, Org. Lett., 2011, 13, 3182; (b) G. H. Chen, J. Y. Gui, L. C. Li and J. Liao, Angew. Chem., Int. Ed., 2011, 50, 7681; (c) Z. Q. Liu, X. Q. Feng and H. F. Du, Org. Lett., 2012, 14, 3154; (d) X. Feng and H. Du, Asian J. Org. Chem., 2012, 1, 204; (e) H. Wang, T. Jiang and M. H. Xu, J. Am. Chem. Soc., 2013, 135, 971; $(f)$ F. Xue, C. G. Li, J. Chen and B. S. Wan, Chin. J. Org. Chem., 2014, 34, 267; $(g)$ Y. Li and M. H. Xu, Chem. Commun., 2014, 50, 3771; (h) Y. Li, Y. N. Yu and M. H. Xu, ACS Catal., 2016, 6, 661; (i) Y. F. Zhang, D. Chen, W. W. Chen and M. H. Xu, Org. Lett., 2016, 18, 2726; (j) T. Jiang, W. W. Chen and M. H. Xu, Org. Lett., 2017, 19, 2138; (k) C. Y. Wu, Y. F. Zhang and M. H. Xu, Org. Lett., 2018, 20, 1789; (l) Y. Li, B. Liu and M. H. Xu, Org. Lett., 2018, 20, 2306.

13 Z. G. Wang, C. G. Feng, S. S. Zhang, M. H. Xu and G. Q. Lin, Angew. Chem., Int. Ed., 2010, 49, 5780.

14 (a) T. Hayashi, M. Takahashi, Y. Takaya and M. Ogasawara, J. Am. Chem. Soc., 2002, 124, 5052; (b) T. Hayashi, K. Ueyama, N. Tokunaga and K. Yoshida, J. Am. Chem. Soc., 2003, 125, 11508. 